\title{
Comparación de dos métodos de obtención de índices para la estimación del riesgo de lixiviación de plaguicidas en dos perfiles de suelo
}

\author{
Bedmar, F.; J.L. Costa; D. Gimenez y P. Daniel
}

\begin{abstract}
RESUMEN
La determinación de los parámetros de adsorción y disipación de plaguicidas en el suelo permite un mejor pronóstico de los procesos y del potencial de contaminación del agua subterránea. El objetivo fue comparar dos métodos de cálculo de los índices factor de retardo y de atenuación log-transformado (AFT), a fin de estimar el riesgo de lixiviación potencial de los herbicidas atrazina, acetoclor y s-metolacloro en los horizontes A, B, C y en todo el perfil de dos suelos de la provincia de Buenos Aires (Argentina). Se elaboraron los índices a partir de los parámetros de adsorción y persistencia de los herbicidas obtenidos mediante experimentos con enfoque multicapa, respecto de cálculos mediante bases de datos con enfoque monocapa. Se determinó el índice de adsorción y el tiempo de vida media para los tres horizontes, a partir de experimentos bajo condiciones controladas. De acuerdo al AFT, calculado para diferentes recargas $(0,3 ; 1,1$ y 2,3 mm/día) mediante información de los experimentos multicapa, se estimó un potencial de lixiviación muy improbable de los tres herbicidas en todos los horizontes y perfiles. La utilización de índices tabulados determinó un mayor riesgo de lixiviación, que tendió a incrementarse a mayor profundidad según el orden $\mathrm{C}>\mathrm{B}>\mathrm{A}>$ perfil.
\end{abstract}

Palabras clave: herbicidas, agua subterránea, factor de retardo, factor de atenuación, lixiviación potencial.

Bedmar, F.; J.L. Costa; D. Gimenez and P. Daniel, 2013. Comparison of two methods for obtaining indices to estimate the risk of leaching of pesticides into two soil profiles. Agriscientia 30 (2): 69-78

\section{SUMMARY}

The determination of adsorption and dissipation parameters of pesticides in soils allows for better prediction of their behavior and potential for groundwater contamination. The objective of this study was to compare two methods of estimating the indices for retardation factor and for attenuation factor logtransformed (AFT). The indices were applied to estimate lixiviation risks of the herbicides acetochlor, atrazine and s-metolachlor in the horizons A, B, C and the whole profile for two soils from Buenos Aires province (Argentina). The 
comparison of the methods consisted in estimating the indices using adsorption and persistence of herbicides obtained from batch experiments (multilayer setting) and from databases (monolayer setting). Herbicide sorption (Koc) and half-life $\left(T_{1 / 2}\right)$ were determined for horizons $A$, B and $C$ from controlled batch experiments. Estimates of AFT calculated from batch experiments for different recharge rates $(0.3,1.1$ and $2.3 \mathrm{~mm} /$ day), resulted in very unlikely leaching potentials for the three herbicides in all horizons and soil profiles. However, using tabulated indices it was determined an increased leaching risk, which increased with increasing depth in the following order $\mathrm{C}>\mathrm{B}>\mathrm{A}>$ profile.

Key words: herbicides, groundwater, retardation factor, attenuation factor, leaching potential.

F. Bedmar y P. Daniel: Facultad de Ciencias Agrarias, Universidad Nacional de Mar del Plata. C.C. 276, C.P. 7620. Balcarce, Buenos Aires, Argentina. J.L. Costa: EEA Balcarce - INTA, Buenos Aires, Argentina. D. Gimenez: Department of Environmental Sciences, Rutgers, The State University of New Jersey, New Brunswick, New Jersey, USA. Correspondencia a: bedmar.francisco@inta.gob.ar

\section{INTRODUCCIÓN}

Atrazina, acetoclor y s-metolaclor son tres de los herbicidas más utilizados en la Argentina para el control de malezas en diversos cultivos, que se aplican usualmente en mezclas que incluyen atrazina con alguno de los otros, a fin de complementar dicho control y abarcar un mayor espectro de acción (Casafe, 2009). La presencia de atrazina en el agua subterránea ha sido motivo de referencia frecuente, tanto en el caso del compuesto parental como de sus metabolitos, especialmente dietilatrazina y deisopropilatrazina (Kolpin et al., 1997; Barbash et al., 2001; Hancock et al., 2008; Steele et al.; 2008). También ha sido comunicada la presencia en el agua subterránea, de acetoclor y metolacloro y de sus metabolitos de degradación (ácido oxanílico, OA, y etanosulfónico, ESA) (De Guzmán et al., 2005; Hackett et al.; 2005; Hancock et al., 2008; Steele et al., 2008).

La predicción del comportamiento de los plaguicidas liberados en el ambiente es necesaria para anticipar, y por ende minimizar, impactos adversos fuera del punto de aplicación (Wagenet \& Rao, 1990). Esto implica que se debe comprender qué le sucede a un plaguicida que ha sido aplicado en el campo y predecir su destino en el ambiente. Utilizando esta información, pueden estimarse los probables impactos adversos sobre el agua superficial o subterránea y/o sobre la salud humana.

Se han propuesto diversos índices, así como modelos simples de investigación preliminar, para determinar el potencial relativo de lixiviación de los plaguicidas a través de la zona de exploración de las raíces de los cultivos. Entre ellos se encuentran el factor de atenuación (AF) y el factor de retardo (RF) (Rao et al., 1985), que se calculan a partir de parámetros de adsorción y de degradación de los plaguicidas, la recarga de agua y otros parámetros físicos de los suelos para una profundidad establecida. Estos índices permiten estimar la contaminación potencial del agua subterránea basada en el riesgo de lixiviación de los plaguicidas. El AF estima la fracción de plaguicida aplicado a la superficie que lixivia a través del perfil del suelo, mientras que el RF determina la movilidad de los plaguicidas a través de éste.

La mayoría de los trabajos utiliza información de índices de adsorción y de persistencia de los plaguicidas proveniente de bases de datos (Díaz-Díaz et al., 1999; Paraiba \& Spadotto, 2002; Paraiba et al., 2003; Kookana et al., 2005; de Paz \& Rubio, 2006; Gianelli et al., 2010). Sin embargo, pocos autores han evaluado el riesgo de lixiviación potencial con los índices RF y AF aplicando valores obtenidos bajo las condiciones ambientales locales (Díaz-Díaz \& Loague, 2000; Bernard et al., 2005). Esto es debido a que la obtención de información local de los parámetros de adsorción y persistencia de gran cantidad de plaguicidas en muchos suelos o situaciones ambientales, tornaría la experimentación en muchos casos impracticable.

Cuando se busca realizar estimaciones con pocos plaguicidas y ambientes, un detalle 
pormenorizado de sus parámetros de adsorción y persistencia podría mejorarlas, reduciendo la variabilidad producida al utilizar información no específica. Si el objetivo es realizar comparaciones múltiples del riesgo de contaminación con plaguicidas, especialmente a escala regional, la utilización de bases de datos ofrece una versión inicial de la situación que luego podría ser estudiada con mayor profundidad. La forma de obtener los índices de adsorción y de degradación se relaciona con las escalas de estudio y, por lo tanto, con diferentes niveles de detalle requerido. Según Loague (1994) el uso de índices provenientes de bases de datos debería considerarse sólo para realizar estudios iniciales de selección y no como método cuantitativo o predictivo para determinar la lixiviación de los plaguicidas.

Por otra parte, en muchos casos el cálculo del AF y RF se realiza considerando al perfil de suelo homogéneo (enfoque monocapa), es decir, se asume que las propiedades del suelo y los índices de adsorción (Koc) y de vida media $\left(T_{1 / 2}\right)$ son invariables a través del perfil. En general, en el perfil de suelo el contenido de carbono orgánico, la densidad de las poblaciones microbianas y su actividad disminuyen significativamente con la profundidad (Skipper et al., 1996) afectando de esta manera la adsorción, degradación y movimiento de los herbicidas (Jenks et al., 1998; Bedmar et al., 2011). Estas variaciones que afectan la magnitud de la adsorción y de la degradación de los herbicidas con la profundidad, debieran ser incorporadas en el cálculo de AF y RF. En contraposición, se ha planteado que el cálculo de los índices se realice individualmente para capas de suelo dentro del perfil. Los índices resultantes en cada capa posteriormente se integran a través de un factor multiplicativo a fin de poder determinar los índices globales del perfil de suelo (enfoque multicapa) (Di et al., 1995; Paraiba \& Spadotto, 2002; Spadotto et al., 2002; Kookana et al., 2005). Si bien este enfoque es conceptualmente más correcto que la consideración de un perfil homogéneo, debe tenerse en cuenta también que la división del perfil en varias capas no garantiza que sus propiedades sean homogéneas, debido a que podrían existir transiciones entre horizontes que estuviesen comprendidas dentro de la misma capa. Es por ello que un enfoque de cálculo por horizontes mejoraría sustancialmente la confiabilidad de los resultados.

El objetivo del trabajo fue comparar dos métodos de cálculo de los factores de atenuación y de retardo, a fin de estimar el riesgo de lixiviación potencial de atrazina, acetoclor y s-metolacloro en dos perfiles de suelo.

\section{MATERIALES Y MÉTODOS}

\section{Área de estudio, suelos y propiedades de los herbicidas}

Los suelos se obtuvieron de dos lugares bajo siembra directa en la provincia de Buenos Aires, Argentina. El primer sitio, General Alvarado (GA), está localizado en el sudeste de la provincia de Buenos Aires ( $38^{\circ} 21^{\prime} \mathrm{S}, 58^{\circ} 07^{\prime} \mathrm{O}$; $13 \mathrm{~m} \mathrm{snm}$ ); mientras que el segundo, Tres Arroyos (TA), está en el sur de la misma provincia $\left(38^{\circ} 44^{\prime} \mathrm{S}, 58^{\circ}\right.$ 44' O; $97 \mathrm{~m} \mathrm{snm).} \mathrm{El} \mathrm{clima} \mathrm{presenta} \mathrm{un} \mathrm{régimen}$ hídrico subhúmedo-seco, con precipitaciones anuales superiores a los $700 \mathrm{~mm}$, distribuidas principalmente entre primavera y otoño, que decrecen de este a oeste. Las temperaturas mínimas y máximas medias anuales en GA son 3 ${ }^{\circ} \mathrm{C}$ y $30^{\circ} \mathrm{C}$, mientras que en TA son $3^{\circ} \mathrm{C}$ y $28^{\circ} \mathrm{C}$, respectivamente. El suelo en GA es un Argiudol típico de la serie Necochea (fino, limoso, mixto), mientras que el suelo de TA es Argiudol típico de la serie Tres Arroyos (fino, ilítico, térmico) (USDA, 2010). El material parental de ambos suelos es loess, y los perfiles están bien a moderadamente drenados. Ambos lugares poseen pendientes suaves $(0 \%-1 \%)$ y capas freáticas relativamente profundas (4 m y 6 m en GA y TA, respectivamente). Las principales rotaciones de cultivos en ambos lugares combinan trigo o maíz con girasol o soja.

El muestreo y extracción de las muestras de suelo en cada lugar se realizó en tres calicatas excavadas hasta debajo del horizonte C. En cada calicata se obtuvieron muestras de suelo de los horizontes A, B y C para determinar las propiedades físicas y químicas (Tabla 1). Todas las muestras de suelo obtenidas para caracterizar las propiedades químicas y algunas físicas, se secaron a $30^{\circ} \mathrm{C}$ y se tamizaron a $2 \mathrm{~mm}$ de malla.

Las propiedades de las muestras de suelo se determinaron según la siguiente metodología:

a) textura: se realizó por el método de la pipeta para determinar las fracciones de los tamaños de partículas por deposición gravimétrica (Gee \& Bauder, 1986); b) carbono orgánico (CO): se analizó mediante oxidación con ácido crómico (Walkley \& Black, 1934); c) pH: se midió con electrodo en una relación suelo:agua de 1:2.5; d) capacidad de intercambio catiónico (CIC): se determinó por la sustitución con acetato de amonio $1 \mathrm{M} \mathrm{y} \mathrm{pH=}$ 7 (Chapman, 1965); e) densidad aparente $\left(\rho_{\mathrm{b}}\right)$ : se estimó a partir de seis columnas no disturbadas (6 $\mathrm{cm}$ de altura y $8,5 \mathrm{~cm}$ de diámetro) de cada horizonte secadas a $105{ }^{\circ} \mathrm{C}$; f) capacidad de campo: se estimó para cada horizonte sobre tres 
Tabla 1. Propiedades físicas y químicas de los horizontes A, B y C de los suelos de General Alvarado (GA) y Tres Arroyos (TA).

\begin{tabular}{|c|c|c|c|c|c|c|c|c|c|c|}
\hline Suelo & Horizonte & $\begin{array}{c}\text { Profundidad } \\
\mathrm{cm}\end{array}$ & $\begin{array}{c}\mathrm{CO} \\
\% \\
\end{array}$ & $\mathrm{pH}$ & $\begin{array}{c}\mathrm{CIC} \\
\mathrm{Cmol} \mathrm{kg}^{-1}\end{array}$ & $\begin{array}{c}\rho_{\mathrm{d}} \\
\mathrm{g} \mathrm{cm}^{-3}\end{array}$ & $\begin{array}{c}\theta_{\mathrm{CC}} \\
\mathrm{cm}^{3} \mathrm{~cm}^{-3}\end{array}$ & Arcilla & $\begin{array}{c}\text { Limo } \\
\% \\
\end{array}$ & Arena \\
\hline \multirow[t]{3}{*}{ GA } & $A$ & $0-41$ & 3,74 & 5,9 & 28,7 & 1,16 & 27,1 & 25,0 & 42,5 & 32,5 \\
\hline & B & $41-81$ & 1,35 & 6,7 & 29,5 & 1,29 & 30,6 & 30,9 & 37,2 & 31,9 \\
\hline & C & $81-130$ & 0,23 & 7,3 & 19,5 & 1,30 & 23,6 & 15,9 & 40,2 & 43,9 \\
\hline \multirow[t]{3}{*}{ TA } & $A$ & $0-26$ & 1,85 & 5,6 & 18,4 & 1,38 & 21,0 & 18,0 & 35,9 & 46,1 \\
\hline & B & $26-51$ & 1,23 & 6,1 & 20,3 & 1,31 & 23,8 & 23,9 & 32,9 & 43,2 \\
\hline & C & $51-110$ & 0,31 & 6,4 & 17,5 & 1,30 & 19,7 & 16,5 & 34,1 & 49,4 \\
\hline
\end{tabular}

$\overline{\mathrm{pH}}, \mathrm{pH}$ suelo/agua 1:2,5; CO, contenido de carbono orgánico; CIC, capacidad de intercambio catiónico; $\rho_{\mathrm{d}}$, densidad aparente; $\theta_{\mathrm{cC}}$, contenido volumétrico de agua a capacidad de campo.

repeticiones mediante la metodología de las ollas de presión utilizando una tensión de 10,33, 500 y 1500 kPa (Klute \& Dirksen, 1986); g) índices de adsorcion (Koc) y de persistencia $\left(T_{1 / 2}\right)$ : los indices usados para el calculo de RF y AF para cada perfil y cada horizonte se encuentran publicados en Bedmar (2009).

\section{Análisis (cuantificación)}

Los extractos de suelo fueron analizados mediante un cromatógrafo Hewlett Packard 1100 HPLC System (Agilent Technologies, HewlettPackard Strasse 8, 76337 Waldbronn, Germany), consistente en un sistema de bomba cuaternaria, automuestreador, horno de columna y detector de arreglo de diodos (DAD). Se utilizó una columna análitica para HPLC Synergi Max RP C12 (Phenomenex, Corporate Headquarters, 411 Madrid Avenue, Torrance, CA, 90501-1430, USA) de $150 \times$ 4,6 mm, Cat. OOF-4337-EO PHENOMENEX. Las longitudes de onda utilizadas para las lecturas de los herbicidas fueron $224 \mathrm{~nm}$ para atrazina y $196 \mathrm{~nm}$ para acetoclor y s-metolacloro. La fase móvil utilizada fue de 1,2 $\mathrm{mL} / \mathrm{min}$ de una solución tampón (95\% 0,025M Na $\mathrm{H}_{2} \mathrm{PO}_{4}, \mathrm{pH}=5,0+5 \%$ acetonitrilo) al $100 \%$ durante 1 min seguido por un gradiente de 22 min hasta alcanzar 40\% de acetonitrilo (Townsend, 1999).

\section{Factor de atenuación y de retardo}

El factor de atenuación (AF) (Rao et al., 1985) se define como la fracción de la masa inicial de herbicida aplicada a la superficie que lixiviará a través de una determinada profundidad del perfil del suelo, y se expresa a través de la siguiente ecuación:

$$
A F=\exp \left[-\left(0.693 d \theta_{C C} R F\right) /\left(q^{T} T_{1 / 2}\right)\right]
$$

donde $\mathrm{d}(\mathrm{cm})$ es la profundidad del horizonte de suelo considerado, $\theta_{\mathrm{CC}}\left(\mathrm{m}^{3} / \mathrm{m}^{3}\right)$ es el contenido volumétrico de agua en el suelo a capacidad de campo, q (cm/día) es la recarga neta de agua subterránea (Aparicio et al., 2008), y $T_{1 / 2}$ (días) es la vida media de los herbicidas en el suelo. El término RF es el factor de retardo (RF), el cual indica la movilidad relativa de un plaguicida que lixivia a través del suelo como consecuencia de la adsorción y la partición entre las fases sólidas y líquidas. Para productos no volátiles, RF se define como

$$
\mathrm{RF}=1+\left(\rho_{\mathrm{d}} \mathrm{foc} \mathrm{Koc}\right) /\left(\theta_{\mathrm{CC}}\right)
$$

donde $\rho_{d}\left(\mathrm{Mg} \mathrm{m}^{-3}\right)$ es la densidad aparente del suelo, foc (\%) es la fracción de carbono orgánico, $\operatorname{Koc}\left(L \mathrm{~kg}^{-1}\right)$ es el coeficiente de partición en carbono orgánico.

Para construir tanto el AF como el RF, se consideraron las propiedades y profundidad (d) de los distintos horizontes que componen el perfil de los suelos, así como se utilizaron los coeficientes de adsorción y de vida media de los herbicidas (Tabla 2). Una vez obtenidos los factores para cada horizonte, se determinaron los factores globales para el perfil de suelo, mediante la integración de los valores de cada horizonte. Así, el factor de retardo o atenuación global del perfil completo se obtuvo multiplicando los valores individuales de acuerdo a la ecuación (Spadotto et al., 2002; Kookana et al., 2005):

$\mathrm{RF} \circ \mathrm{AF}$ global $=\mathrm{RF} \circ \mathrm{AF}$ horiz. $\mathrm{A} \times \mathrm{RF} \circ \mathrm{AF}$ horiz. B X RF o AF horiz. C

Asimismo, debido a la posible ocurrencia de valores muy pequeños y con elevados desvíos del índice $A F$, se utilizó la transformación propuesta por Li et al. (1998), la cual permite obtener el índice AFT:

$$
\mathrm{AFT}=\operatorname{Ln}(\mathrm{AF}) /(-0,693)
$$

donde $\operatorname{Ln}(\mathrm{AF})$ es el logaritmo natural del factor de atenuación y -0,693 es el Ln de 0,5 (mitad del tiempo).

De acuerdo a lo establecido anteriormente, 
Tabla 2, Indices de adsorción ( $\mathrm{Kd}, \mathrm{Koc}$ ) y persistencia $\left(T_{1 / 2}, \mathrm{k}\right)$ a $20^{\circ} \mathrm{C}$, para acetoclor, atrazina y s-metolacloro en los horizontes A, B y C de dos suelos ${ }^{1}$ (GA: General Alvarado; TA: Tres Arroyos) (Bedmar, 2009) y de bases de datos (Senseman, 2007).

\begin{tabular}{|c|c|c|c|c|c|c|}
\hline Herbicida & Suelo & Horizonte & $\mathrm{Kd}$ & $\mathrm{L} \mathrm{kg}^{-1}$ & $\begin{array}{l}T_{1 / 2} \\
\text { días }\end{array}$ & $\begin{array}{c}k \\
d^{-1}\end{array}$ \\
\hline \multirow[t]{7}{*}{ acetoclor } & GA & A & $8,2(0,6)$ & $220(16)$ & $21,2(3,1)$ & $0,0368(0,0046)$ \\
\hline & & B & $4,4(1,0)$ & $330(48)$ & $30,3(13,0)$ & $0,0254(0,0087)$ \\
\hline & & C & $4,3(0,5)$ & 2570 1606) & $61,8(14,0)$ & $0,0116(0,0023)$ \\
\hline & TA & A & $5,2(0,3)$ & $283(21)$ & $25,4(2,4)$ & $0,0277(0,0025)$ \\
\hline & & B & $5,0(0,4)$ & $412(20)$ & $40,2(8,7)$ & $0,0178(0,0038)$ \\
\hline & & C & $4,7(0,4)$ & 2209 (1894) & $75,4(2,1)$ & $0,0092(0,0003)$ \\
\hline & & base de datos & - & 130 & 13 & - \\
\hline \multirow[t]{7}{*}{ atrazina } & GA & A & $9,3(0,5)$ & $259(51)$ & $16,0(4,7)$ & $0,0487(0,0148)$ \\
\hline & & B & $5,4(0,5)$ & 409 (59) & $18,1(3,1)$ & $0,0391(0,0067)$ \\
\hline & & C & $4,2(0,2)$ & 2620 (1718) & $25,4(6,2)$ & $0,0283(0,0062)$ \\
\hline & TA & A & $7,4(0,4)$ & $398(23)$ & $14,0(2,8)$ & $0,0505(0,0019)$ \\
\hline & & B & $5,6(0,2)$ & $460(22)$ & $18,3(2,8)$ & $0,0386(0,0063)$ \\
\hline & & C & $4,7(0,6)$ & 2451 (2528) & $28,6(8,7)$ & $0,0255(0,0066)$ \\
\hline & & base de datos & - & 100 & 60 & - \\
\hline \multirow[t]{7}{*}{ s-metolacloro } & GA & A & $21,8(1,0)$ & $596(70)$ & $98,5(19,4)$ & $0,0074(0,0015)$ \\
\hline & & B & $20,3(1,5)$ & 1556 (396) & $115,0(35,5)$ & $0,0064(0,0019)$ \\
\hline & & C & $13,1(1,5)$ & 8259 (5869) & $126,4(14,9)$ & $0,0055(0,0007)$ \\
\hline & TA & A & $17,3(1,6)$ & $938(64)$ & $87,8(7,8)$ & $0,0080(0,0008)$ \\
\hline & & B & $13,7(1,2)$ & $1120(42)$ & $132,0(8,3)$ & $0,0053(0,0003)$ \\
\hline & & C & $11,9(0,5)$ & $5803(5351)$ & $143,1(11,7)$ & $0,0049(0,0004)$ \\
\hline & & base de datos & - & 200 & 90 & - \\
\hline
\end{tabular}

${ }^{1}$ valores expresados como promedio y entre paréntesis desvío estándar.

el factor de retardo (RF) y de atenuación Lntransformado (AFT) fueron calculados para los horizontes A, B y C, así como para el perfil completo de los suelos de GA y TA, considerando un enfoque multicapa o monocapa. En el caso del enfoque multicapa, se utilizaron los valores del coeficiente de partición en carbono orgánico de los herbicidas (Koc) y la vida media ( $T_{1 / 2}$ ) obtenidos para cada horizonte, a partir de los experimentos de adsorción y de persistencia (Tabla 2), los cuales fueron integrados por medio de la ecuación (3) para obtener los factores del perfil. En cuanto al enfoque monocapa, se utilizaron los valores de Koc y $T_{1 / 2}$ provenientes de base de datos (Tabla 2) y considerados en forma similar para todos los horizontes para el cálculo de los factores del perfil (ecuación 3).

A fin de realizar evaluaciones y comparaciones relativas de la movilidad y lixiviación potencial de los herbicidas mediante los índices RF y AFT, se utilizó la clasificación de Bernard et al. (2005) (Tabla 3).

Tabla 3, Escalas relativas de movilidad herbicida y potencial de lixiviación (Bernard et al., 2005), para el factor de retardo (RF), factor de atenuación (AF) y el factor de atenuación In-transformado (AFT).

\begin{tabular}{lrccc}
\hline \multicolumn{1}{r}{ RF } & Clasificación & AF & AFT & Clasificación \\
\hline$=1$ & muy móvil & $\geq 2,5 \times 10^{-1} \mathrm{y} \leq 1$ & $\leq 2$ & muy probable \\
$>1 \mathrm{y}<2$ & móvil & $\geq 1 \times 10^{-1} \mathrm{y}<2,5 \times 10^{-1}$ & $\geq 2 \mathrm{y}<3$ & probable \\
$\geq 2 \mathrm{y}<3$ & moderadamente móvil & $\geq 1 \times 10^{-2} \mathrm{y}<1 \times 10^{-1}$ & $\geq 3,3 \mathrm{y}<7,2$ & moderadamente probable \\
$\geq 3 \mathrm{y}<10$ & moderadamente inmóvil & $\geq 1 \times 10^{-4} \mathrm{y}<1 \times 10^{-2}$ & $\geq 7,2 \mathrm{y}<13,3$ & improbable \\
$\geq 10$ & muy inmóvil & $<1 \times 10^{-4}$ & $>13,3$ & muy improbable \\
\hline
\end{tabular}




\section{RESULTADOS Y DISCUSIÓN}

Los valores de RF calculados a partir del Koc experimental obtenido para cada horizonte (Tabla 4), indicaron que los tres herbicidas se encuadraron como "muy inmóviles" ( $\geq 10)$, tanto en los horizontes como en el perfil. Por el contrario, los valores de RF calculados a partir de los Koc de base de datos fueron menores a los obtenidos a partir de Koc experimental (Tabla 5). En tal sentido, para ambos sitios se comprobó que tanto atrazina como acetoclor calificaron como "moderadamente inmóviles" ( $\geq 3$ y $<10$ ) en el horizonte $B$, al igual que s-metolacloro en el horizonte $\mathrm{C}$. Por su parte, atrazina y acetoclor resultaron "moderadamente móviles" ( $\geq 2<3$ ) en el horizonte C de GA, mientras que acetoclor en el horizonte $C$ de TA fue "moderadamente inmóvil" ( $\geq 3$ y $<10$ ). Al igual que para el cálculo por medio del Koc experimental, los tres herbicidas se clasificaron como "muy inmóviles" al considerar el perfil de los suelos.

En lo referente al factor de atenuación Lntransformado (AFT), en la Figura 1 se presentan los resultados obtenidos para tres valores de recarga neta ( $q=0,3 ; 1,1$ y 2,3 $\mathrm{mm} /$ día). Según los resultados, se determinó que tanto cuando se realizaron los cálculos con los valores de Koc y vida media $\left(T_{1 / 2}\right)$ obtenidos para cada horizonte, como cuando se utilizaron estas variables a partir de la base de datos disponibles, los tres herbicidas incrementaron su potencial de lixiviación con el aumento de la recarga neta (q), tanto en los horizontes como en el perfil de los suelos (Figura 1). Sin embargo, al considerar los Koc y $\mathrm{T}_{1 / 2}$ calculados para cada horizonte, todos los herbicidas presentaron valores de AFT superiores a 13,3 (Tabla 4), por lo cual reportaron un potencial de lixiviación "muy improbable" en todos los horizontes, perfiles y recargas consideradas para ambos sitios (GA y TA) (Figura 1).

En lo concerniente al factor de atenuación (AFT) calculado según valores de Koc y $T_{1 / 2}$ provenientes de base de datos (Figura 1), los valores y tendencias obtenidas difirieron según la recarga, el sitio, el horizonte considerado y el herbicida. En este sentido, tanto en GA como en TA, los tres herbicidas presentaron riesgo de lixiviación "muy improbable" (AFT > 13,3) para la menor recarga $(0,3$ $\mathrm{mm} /$ día). Sin embargo, al incrementarla a $1,1 \mathrm{~mm} /$ día, sólo atrazina y s-metolacloro mostraron riesgo de lixiviación entre "moderadamente probable" e "improbable" en los horizontes B y C de ambos suelos. Finalmente, cuando la recarga fue máxima (2,3 $\mathrm{mm} /$ día), tanto atrazina como s-metolacloro exhibieron riesgo de lixiviación "probable" en el horizonte C de GA y TA, y entre "probable" y "moderadamente probable" en el horizonte B de ambos suelos, mientras que acetoclor sólo mostró riesgo "improbable" en el horizonte $\mathrm{C}$ de GA. En lo referente al comportamiento en el perfil de suelo, con la excepción de atrazina en el sitio TA, que resultó "improbable", todos los herbicidas presentaron riesgos "muy improbables" de lixiviación según la clasificación de riesgo utilizada.

Tabla 4. Factor de retardo (RF) de atrazina, acetoclor y s-metolacloro en los horizontes A, B y C de suelos de General Alvarado (GA) y Tres Arroyos (TA). Cálculos realizados considerando los valores del coeficiente de partición en carbono orgánico (Koc) obtenidos para cada horizonte (enfoque multicapa).

\begin{tabular}{lcccccccc}
\hline & \multicolumn{9}{c}{ GA } & \multicolumn{3}{c}{ TA } \\
\cline { 2 - 8 } \multicolumn{1}{c}{ Herbicida } & A & B & C & Perfil & A & B & C & Perfil \\
\hline atrazina & $42,1(5,8)$ & $24,1(3,1)$ & $24,2(1,9)$ & $>100$ & $49,3(4,2)$ & $32,1(1,5)$ & $27,0(5,6)$ & $>100$ \\
acetoclor & $36,1(1,7)$ & $19,7(3,1)$ & $24,5(3,6)$ & $>100$ & $35,2(1,2)$ & $28,9(2,8)$ & $28,3(10,2)$ & $>100$ \\
s-metolacloro & $95,8(5,9)$ & $89,0(21,1)$ & $72,7(6,1)$ & $>100$ & $>100$ & $77,0(8,2)$ & $68,8(21,6)$ & $>100$ \\
\hline
\end{tabular}

Tabla 5. Factor de retardo (RF) de atrazina, acetoclor y s-metolacloro en los horizontes A, B y C de suelos de General Alvarado (GA) y Tres Arroyos (TA). Cálculos realizados considerando valores del coeficiente de partición en carbono orgánico (Koc) obtenidos de bases de datos y similares para todos los horizontes (enfoque monocapa).

\begin{tabular}{lcccccccc}
\hline & \multicolumn{9}{c}{ GA } & \multicolumn{4}{c}{ TA } \\
\cline { 2 - 8 } \multicolumn{1}{c}{ Herbicida } & A & B & C & Perfil & A & B & C & Perfil \\
\hline atrazina & $17,0(0,9)$ & $6,7(0,2)$ & $2,3(1,1)$ & $>100$ & $13,1(0,5)$ & $7,8(0,6)$ & $3,1(1,5)$ & $>100$ \\
acetoclor & $21,8(1,1)$ & $8,4(0,3)$ & $2,7(1,5)$ & $>100$ & $16,8(0,7)$ & $9,8(0,8)$ & $3,7(2,0)$ & $>100$ \\
s-metolacloro & $33(1,8)$ & $12,3(0,4)$ & $3,7(2,2)$ & $>100$ & $25,3(1,0)$ & $14,6(1,2)$ & $5,2(3,1)$ & $>100$ \\
\hline
\end{tabular}

a valores en negrita y cursiva indican que, considerando el desvío estándar (entre paréntesis), RF < 10. 

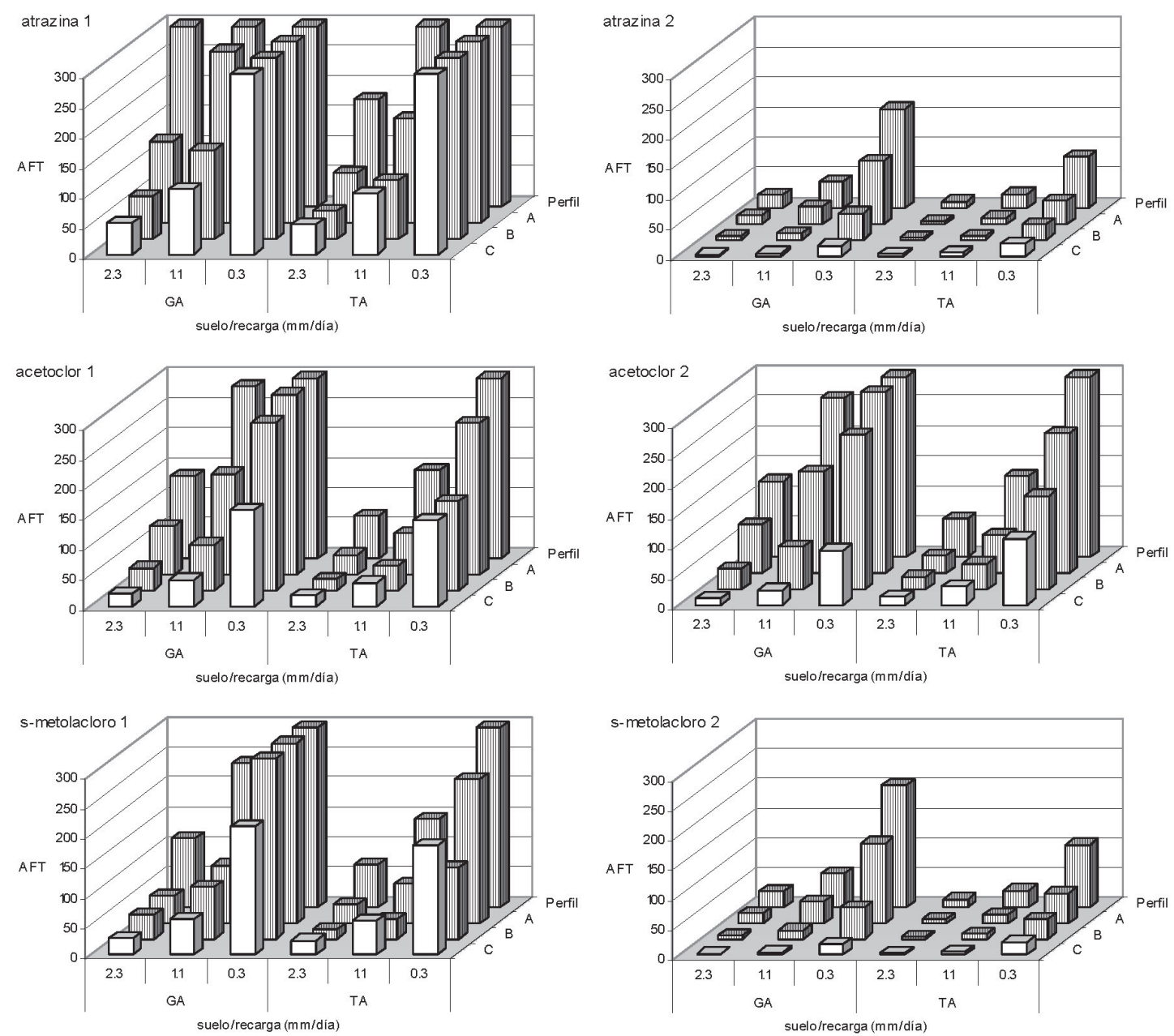

Figura 1. Factor de atenuación Ln-transformado (AFT) de atrazina, acetoclor y s-metolacloro, en los horizontes A, B, C y perfil de suelos de General Alvarado (GA) y Tres Arroyos (TA) calculado para tres recargas (0,3,1,1 y 2,3 mm/día). Cálculos para atrazina 1, acetoclor 1 y s-metolacloro 1, realizados con el coeficiente de partición en carbono orgánico (Koc) y la vida media ( $T_{1 / 2}$ ) obtenidos para cada horizonte (enfoque multicapa). Cálculos para atrazina 2, acetoclor 2 y s-metolacloro 2, realizados con el coeficiente de partición en carbono orgánico (Koc) y la vida media $\left(\mathrm{T}_{1 / 2}\right)$ obtenidos de bases de datos y similares para todos los horizontes (enfoque monocapa).

Estos resultados son coincidentes con lo encontrado por Gianelli et al. (2010), quienes determinaron que atrazina, acetoclor $y$ s-metolacloro presentaron un potencial de lixiviación calificado como "muy improbable" para el perfil completo de 18 series de suelos, teniendo en cuenta el índice AFT calculado mediante bases de datos y una recarga de 1,1 mm/día. Asimismo, Diaz-Diaz \& Loague (2000) estudiaron el riesgo potencial de lixiviación de los insecticidas carbofuran y ethoprophos en seis órdenes de suelos de Tenerife (España) para una profundidad de 0,25 $\mathrm{m}$ y valores de recarga entre 1,57 y 3,11 $\mathrm{mm} / \mathrm{día}$, y determinaron que los mayores riesgos estuvieron asociados a las mayores recargas $(1,99-3,11 \mathrm{~mm} / \mathrm{día})$. Por su parte, Chatupote \& Panapitukkul (2005) concluyeron que metolacloro incrementó su potencial de lixiviación (AF) cuando la recarga aumentó de 10 a 30 y 60 mm/día. Por su parte, Bernard et al. (2005) estudiaron el efecto de tres recargas (10, 25 y $50 \mathrm{~mm} /$ día) en dos suelos sobre el riesgo de lixiviación de cinco herbicidas, entre ellos atrazina, y encontraron que el AFT disminuyó en todos los casos con el aumento de la recarga, lo que indica valores de mayor riesgo.

La velocidad a la cual lixivian los plaguicidas a través de la zona no saturada o vadosa, y la proporción de plaguicida aplicado que alcanza el 
agua subterránea, están fuertemente relacionadas al flujo promedio de agua (recarga), así como a su variabilidad temporal (Giambelluca et al., 1996). Así, para una misma precipitación anual, se ha demostrado que la lixiviación se incrementa con la variabilidad temporal (Jury \& Gruber, 1989). Por su parte, Giambelluca et al. (1996) indicaron que la variabilidad temporal en la recarga, a nivel anual, mensual y diaria, produce una incertidumbre significativa en el AF.

En el presente trabajo, el índice AF fue calculado para una recarga obtenida a través de una serie de 8 años, de los cuales se seleccionaron los períodos correspondientes a los valores de máxima (2,3 $\mathrm{mm} /$ día $)$ y mínima $(0,3 \mathrm{~mm} /$ día $)$ recarga de agua, y un promedio de los 8 años (1,1 mm/día) (Aparicio et al., 2008), a partir de los cuales se encontró un incremento significativo del riesgo con el aumento de la recarga (Figura 1). Por tanto, se puede establecer que en años lluviosos o en situaciones de sobre riego, se incrementa el riesgo de lixiviación de los herbicidas estudiados. Por el contrario, bajos volúmenes de drenaje, tal como sucedió con la recarga de 0,3 mm/día, aun con elevado contenido de plaguicidas en el suelo conllevarán a una reducción de las cantidades transportadas (Grignani \& Laidlow, 2002).

Los resultados obtenidos muestran que el cálculo de los factores de retardo (RF) y atenuación (AF) varió al utilizar valores de Koc o $T_{1 / 2}$ experimentales o de base de datos. El uso de Koc experimentales generó valores elevados del RF, por lo cual los herbicidas se clasificaron como "muy inmóviles" (Tabla 4), implicando una reducida, sino nula, movilidad de los tres herbicidas, tanto en los horizontes como en el perfil de los suelos de GA y TA. Por el contrario, al realizar los cálculos con Koc de base de datos y enfoque monocapa, se obtuvieron valores de RF que encuadraron a los herbicidas en categorías de mayor movilidad en los horizontes de ambos suelos, no así en el perfil (Tablas 4 y 5 ).

En el caso del AFT, teniendo en cuenta valores experimentales de $\mathrm{Koc}$ y $\mathrm{T}_{1 / 2}$, se determinó un potencial de lixiviación muy improbable de los tres herbicidas en todos los horizontes y perfiles de los suelos de GA y TA para las tres recargas evaluadas. Por el contrario, al considerar para el cálculo valores de base de datos de Koc y $T_{1 / 2}$ similares para los tres horizontes, se estimaron mayores riesgos de lixiviación para todas las recargas.

Los resultados y tendencias observadas se deben primordialmente a que los valores de los coeficientes de adsorción (Koc) obtenidos en laboratorio fueron mayores que los provenientes de base de datos (Tabla 3), situación que incrementa los valores de los factores de retardo y atenuación luego de la resolución de las ecuaciones correspondientes (ecuaciones 1 y 2). Además del efecto del coeficiente de adsorción, el mayor tiempo de vida media $\left(T_{1 / 2}\right)$ obtenido en laboratorio en los casos de acetoclor y s-metolacloro contribuyó parcialmente (Tabla 3), incrementando los valores del AF (ecuación 1). En el caso de atrazina no se presentó una clara relación, debido a que los tiempos de vida media determinados fueron inferiores respecto de los provenientes de base de datos.

A modo de conclusión, puede establecerse que si bien se estimó mayor riesgo de lixiviación mediante el cálculo de los índices RF y AF con índices de adsorción y persistencia de los herbicidas en el suelo proveniente de base de datos y enfoque monocapa, el disponer de coeficientes locales (Koc, $T_{1 / 2}$ ) de los diferentes horizontes del suelo, proveería una base mas confiable para el cálculo de los índices AF y $R F$, dado que supone el reconocimiento de una de las principales características del suelo, su heterogeneidad. Sin embargo, aunque el presente trabajo demuestra que se puede arribar a resultados disímiles según se aborde una $u$ otra forma de cálculo, resulta imprescindible la realización de trabajos experimentales que verifiquen el grado de asociación con la realidad al estimar la vulnerabilidad de los acuíferos por aplicación de plaguicidas.

\section{BIBLIOGRAFIA}

Aparicio, V.; J.L. Costa and M. Zamora, 2008. Nitrate leaching assessment in a long-term experiment under supplementary irrigation in humid Argentina. Agric. Water Manag. 95:1361-1372.

Barbash, J.E.; G.P. Thelin, D.W. Kolpin and R.J. Gilliom, 2001. Major herbicides in ground water: results from the National Water-Quality Assessment. J. Environ. Qual. 30:831-845.

Bedmar, F., 2009. Persistencia, adsorción y transporte de acetoclor, atrazina y s-metolacloro en horizontes superficiales y sub-superficiales de dos Argiudoles Típicos. Tesis Doctoral, FCA-UNMDP, 160 pp.

Bedmar, F.; P. Daniel, J.L. Costa and D. Gimenez, 2011. Sorption of acetochlor, s-metolachlor and atrazine in surface and subsurface soil horizons of Argentina. Environ. Toxicol. Chem. 30:1990-1996.

Bernard, H.; P.F. Chabalier, J.L. Chopart, B. Legube and M. Vauclin, 2005. Assessment of herbicide leaching 
risk in two tropical soils of Reunion Island (France). J. Environ. Qual. 34:534-543.

Casafe, 2009. Guía de Productos Fitosanitarios para la República Argentina. Cámara de Sanidad Agropecuaria y Fertilizantes. $12^{\circ}$ Ed. 1600 pp.

Chapman, H.D., 1965. Cation exchange capacity. In: Black, C.A. (ed.). Methods of soil analysis. American Society of Agronomy, Madison, WI, USA. Agronomy Series No 9:891-900.

Chatupote, W. and N. Panapitukkul, 2005. Regional assessment of nutrient and $\quad \mathrm{pe} \mathrm{s} \mathrm{t} \mathrm{i} \mathrm{c} \mathrm{i} \mathrm{d} \mathrm{e}$ leaching in the vegetable production area of Rattaphum catchment, Thailand. Water Air Soil Pollut. 5:165-173.

De Guzman, N.P.; P. Hendley, D.I. Gustafson, I. Van Wesenbeeck, A.J. Klein, J.D. Fuhrman, K. Travis, N.D. Simmons, W.E. Teskey and R.B. Durham, 2005. The Acetochlor registration partnership state ground water monitoring program. J. Environ. Qual. 34:793-803.

De Paz, J.M. and J.L. Rubio, 2006. Application of a GISAF/RF model to assess the risk of herbicide leaching in a citrus-growing area of the Valencia Community, Spain Sci. Total Environ. 371:44-54.

Di, H.J.; R.S. Kookana and A.G. Aylmore, 1995. Application of a simple model to assess the ground water contamination potential of pesticides. Aust. J. Soil Res. 33:1031-1040.

Díaz-Díaz, R.; K. Loague and J.S. Notario, 1999. An assessment of agrochemicalleaching potentials for Tenerife. J. Contam. Hydrol. 36:1-30.

Diaz-Diaz, R. and K. Loague, 2000. Regional-scale leaching assessments for Tenerife: effect of data uncertainties. J. Environ. Qual. 29:835-847.

Gee, G.W. and J.W. Bauder, 1986. Particle-size analysis. In: Klute, A. (ed.). Methods of soil analysis, Part 1-Physical and mineralogical methods. 2nd ed. American Society of Agronomy and Soil Science Society of America, Madison, WI, USA. Agronomy Series Number 9, Part 1. pp. 383-411.

Giambelluca, T.W.; K. Loague, R.E. Green and M.A. Nullet, 1996. Uncertainty in recharge estimation on groundwater vulnerability assessments for the Pearl Harbor Basin, O'ahu, Hawaii, USA. J. Contam. Hydrol. 23:85-112.

Gianelli, V.; F. Bedmar, H. Angelini, V. Aparicio y J.L. Costa, 2010. Riesgo de contaminación del agua subterránea con plaguicidas en la cuenca del arroyo Pantanoso (R. Argentina). En Contaminación, Descontaminación y Restauración Ambiental en Iberoamérica. Sociedad Iberoamericana de Física y Química Ambiental, Salamanca (España), pp. 135-152.

Grignani, C. and A.S. Laidlow, 2002. Nitrogen economy in grasslands and annual forage crops: control of environmental impact. In Proc. of the $19^{\text {th }}$ General Meeting of the European Grassland Federation on Multifunction Grasslands, Quality Forages, Animal Products and Landscapes British Grassland Society, vol. 7, Reading, UK, La Rochelle (F), pp. 625-633.

Hackett, A.G.; D.I. Gustafson, J.S. Moran, P. Hendley, I. Van Wesenbeeck, N.D. Simmons, A.J. Klein, J.M. Kronenberg, J.D. Fuhrman, J.L. Honegger, J. Hanzas, D. Healy and C. Stone, 2005. The Acetochlor registration partnership surface water monitoring program for four corn herbicides. J. Environ. Qual. 34:877-889.

Hancock, T.C.; M.W. Sandstrom, J.R. Vogel, R.M.T. Webb, E.R. Bayless and J.E. Barbash, 2008. Pesticide fate and transport throughout unsaturated zones in five agricultural settings, USA. J. Environ. Qual. 37:10861100.

Jenks, B.M.; F.W. Roeth, A.R. Martin and D.L. Mccallister, 1998. Influence of surface and subsurface soil properties on atrazine sorption and degradation. Weed Sci. 46:132-138.

Jury, W.A. and J. Gruber, 1989. A stochastic analysis of the influence of soil and climatic variability on the estimate of pesticide groundwater pollution potential. Water Resour. Res. 25:2465-2474. Klute, A. and C. Dirksen, 1986. Hydraulic conductivity and diffusivity: laboratory methods. In: Klute, A. (ed.), Methods of Soil Análisis. Part. 1. ASA and SSSA, Madison, WI, USA. Agronomy No 9, pp. 687-734.

Kolpin, D.W.; D. Sneck-Fahrer, G.R. Hallberg and R.D. Libra, 1997. Temporal trends of selected agricultural chemicals in lowa's groundwater, 1982-1995: Are things getting better?. J. Environ. Qual. 26:1007-1017.

Kookana, R.S.; R.L. Correll and R.B. Miller, 2005. Pesticide impact rating index -a pesticide risk indicator forwater quality. Water Air Soil Pollut. 5:45-65.

Li, Z.C.; R.S. Yost and R.E. Green, 1998. Incorporating uncertainty in a chemical leaching assessment. J. Contam. Hydrol. 29:285-299.

Loague, K., 1994. Regional scale ground water vulnerability estimates: Impact of reducing data uncertainties for assessments in Hawaii. Ground Water. 32:605-616.

OECD (Organisation for Economic Co-operation and Development), 2000. OECD guidelines for the testing of chemicals. Adsorption/desorption using a batch equilibriummethod. OECD Test Guideline, Vol 106. Paris, France.

Paraiba, L.C., and C.A. Spadotto, 2002. Soil temperature effect in calculating attenuation and retardation factors. Chemosphere. 48:905-912.

Paraiba, L.C.; A.L. Cerdeira, E. Fraga Da Silva, J. Souza Martinsand H.L. Da Costa Coutinho, 2003. Evaluation of soil temperature effect on herbicide leaching 
potential into groundwater in the Brazilian Cerrado. Chemosphere. 53:1087-1095.

Rao, P.S.C.; A.G. Hornsby and R.E. Jessup, 1985. Indices for ranking the potential for pesticide contamination of groundwater. Soil Crop Sci. Soc. Florida. Proc. 4:1-8.

Senseman, S.A., 2007. Herbicide handbook. 9th ed. Weed Sci. Soc. of Am., Lawrence, KS, USA. 458 pp.

Skipper, H.D.; A.G. Wollum, R.F. Turco and D.C. Wolf, 1996. Microbiological aspects of environmental fate studies of pesticides. Weed Technol. 10:174-190.

Spadotto, C.A.; M.A.F. Gomes and A.G. Hornsby, 2002. Pesticide leaching potential assessment in multilayered soils. Pesticidas: Revista de Ecotoxicologia e Meio Ambiente. 12:1-12.

Steele, G.B.; H.M. Johnson, M.W. Sandstrom, P.D. Capel and J.E. Barbash, 2008. Occurrence and fate of pesticides in four contrasting agricultural settings in the United States. J. Environ. Qual. 37:1116-1132.

Townsend, I., 1999. Application 1524. HPLC. Application Note. Varian. CP-SCANVIEW for Windows. Version 5.00. Varian Analytical Instruments. Middelburg, The
Netherlands.

USDA, 2010. Claves para la taxonomía de suelos. Undécima edición. Soil Survey Staff, USDA-Natural Resources Conservation Service, Washington, DC, USA, $365 \mathrm{pp}$.

Wagenet, R.J., and P.S.C. Rao, 1990. Modeling pesticide fate in soils. Chapter 10. In Pesticides in the soil Environment: Processes, Impacts, and Modeling. SSSA, Inc. Madison, WI, pp. 351-398.

Walker, A.; E.G. Cotterill and S.J. Welch, 1989. Adsorption and degradation of chlorsulfuron and metsulfuron methyl in soils from different depths. Weed Res. 29:281-287.

Walkley, A. and A.I. Black, 1934. An examination of the Degtjareff method for determining soil organic matter and a proposed modification of the chromic acid titration method. Soil Science. 37:29-37. 